\title{
POLYVINYLPYRROLIDONE COATING FOR NANODIAMOND STABILIZATION IN SALINE SOLUTION AND SILVER NANOPARTICLE DECORATION
}

\author{
${ }^{1}$ Katerina KOLAROVA, ${ }^{1}$ Daria MILIAIEVA, ${ }^{1}$ Stepan STEHLIK \\ ${ }^{1}$ Institute of Physics of the Czech Academy of Sciences, Prague 6, Czech Republic, EU, kolarovak@fzu.cz
}

https://doi.org/10.37904/nanocon.2020.3696

\begin{abstract}
This work presents use of polyvinylpyrrolidone (PVP) for multipurpose coating of oxidized high-pressure hightemperature and detonation nanodiamonds. This simple way of nanodiamonds surface modification aims to improve their colloidal stability in biological environments and reduce their proneness to agglomeration. PVP immobilized on nanodiamond surface also provides for nanodiamond-supported AgNPs preparation by in situ synthesis using $\mathrm{AgNO}_{3}$ as a metallic nanoparticles precursor. Dynamic light scattering (DLS) and UV-vis spectroscopy were used for evaluation of nanoparticles size distribution and dispersibility in water and after exposition of nanoparticles in saline solution. Images acquired by scanning electron microscopy and transmission electron microscope validate the possibility of binding $~ 10 \mathrm{~nm}$ AgNPs to nanodiamonds surface.
\end{abstract}

Keywords: Nanodiamonds, silver nanoparticles, colloid solution

\section{INTRODUCTION}

The diamond nanoparticles (so-called nanodiamonds, NDs) belong to a family of carbon-based nanomaterials The various structure and surface chemistry of ND particles can lead to their numerous applications [1]. Owing to their low chemical reactivity and unique physical properties, nanodiamonds could be useful in a variety of biological applications, novel imaging techniques, coatings for implantable materials and biodetectors [2]. Compared with other similarly sized materials, primary ND particles have a distinct advantage - they are inherently non-toxic; although this property depends on sufficient purification from $\mathrm{sp}^{2}$ carbon, the toxicity also varies depending on the type of cell used.

NDs have been synthesized by various techniques, however the most important and available at industrial scale at the current time is the "bottom-up" method - detonation technique, and the "top-down" - high-energy ball milling of high-pressure high-temperature diamonds (HPHT) [3]. Primary particles of detonation nanodiamonds (DNDs) are single-digit nanometre in size with narrow size distribution and typically spherical in shape. These attributes stem from their origin in a rapid detonation process, which terminates within a fraction of microsecond, severely restricting the time of growth of ND particles. DNDs have diameters of 4-5 $\mathrm{nm}$, but they tend to aggregate and therefore usual commercial suspensions of nanodiamonds contain larger aggregates [1]. DND nanoparticle is modelled by a diamond core, including various lattice defects, transient $\mathrm{sp}^{3} / \mathrm{sp}^{2}$ layer, and $\mathrm{sp}^{2}$ surface shell that may carry various surface functional groups.

Primary HPHT particles are typically sharp and with huge size distribution. Their main advantage over DNDs is their expected uniform structure with low concentration of lattice defects such as dislocations or twinning.

Nanodiamonds have a superior mechanical and thermal properties combined with low cytotoxicity. Moreover, their variable surface chemistry is suited for preparation nanodiamond composites suitable for biomedical composition.

One of the main challenges in preparation of nanodiamond composites is the task of maintaining stability of colloidal solutions and also prevention of re-aggregation of NDs, especially in biological systems [4]. 
The aim of this work is to prepare nanodiamond composites that would be able to maintain their stability and dispersibility even in an unfavorable salt solution environment.

\section{EXPERIMENTAL PART}

The HPHT nanodiamonds (MSY18) were purchased form Microdiamant AG (size range: 0-25nm). The detonation nanodiamonds (DND) were purchased from New Metals and Chemicals Corp. The NDs were oxidized in air at $450^{\circ} \mathrm{C}$ for 5 hours (for MSY18) or at $520^{\circ} \mathrm{C}$ for 50 min for DND nanoparticles. Subsequently the NDs powder was mixed with deionized water (concentration $1 \mathrm{mg} / 1 \mathrm{~mL}$ ) and ultrasonicated by an ultrasound horn for 1 hour. The ND-PVP colloids were prepared as shown in the scheme below (Figure 1). In short - the suspension of annealed nanodiamonds was added to a $2 \% \mathrm{w} / \mathrm{w}$ polyvinylpyrrolidone (PVP K25 from Carl Roth) water solution and stirred at $60^{\circ} \mathrm{C}$. The resulting colloid was overflowed three times by centrifugation to replace the PVP solution with a deionized water. The final ND-PVP colloid in water was used for preparation of silver decorated nanodiamonds. The PVP coated nanodiamond colloid was stirred at $60{ }^{\circ} \mathrm{C}$ and during this time a $2 \% \mathrm{w} / \mathrm{w}$ solution of PVP in ethanol was added into it. Then, silver nitrate was slowly dripped into the mixture (up to a final concertation of $0.1 \mathrm{M} \mathrm{AgNO}_{3}$ ) in a dark place until $\mathrm{Ag}$ nanoparticles formed and the solution turned yellow. During addition of $\mathrm{AgNO}_{3}$, the mixture was still stirred and heated. After 2 hours the silver decorated nanodiamonds were cleaned up by centrifugation as described above.

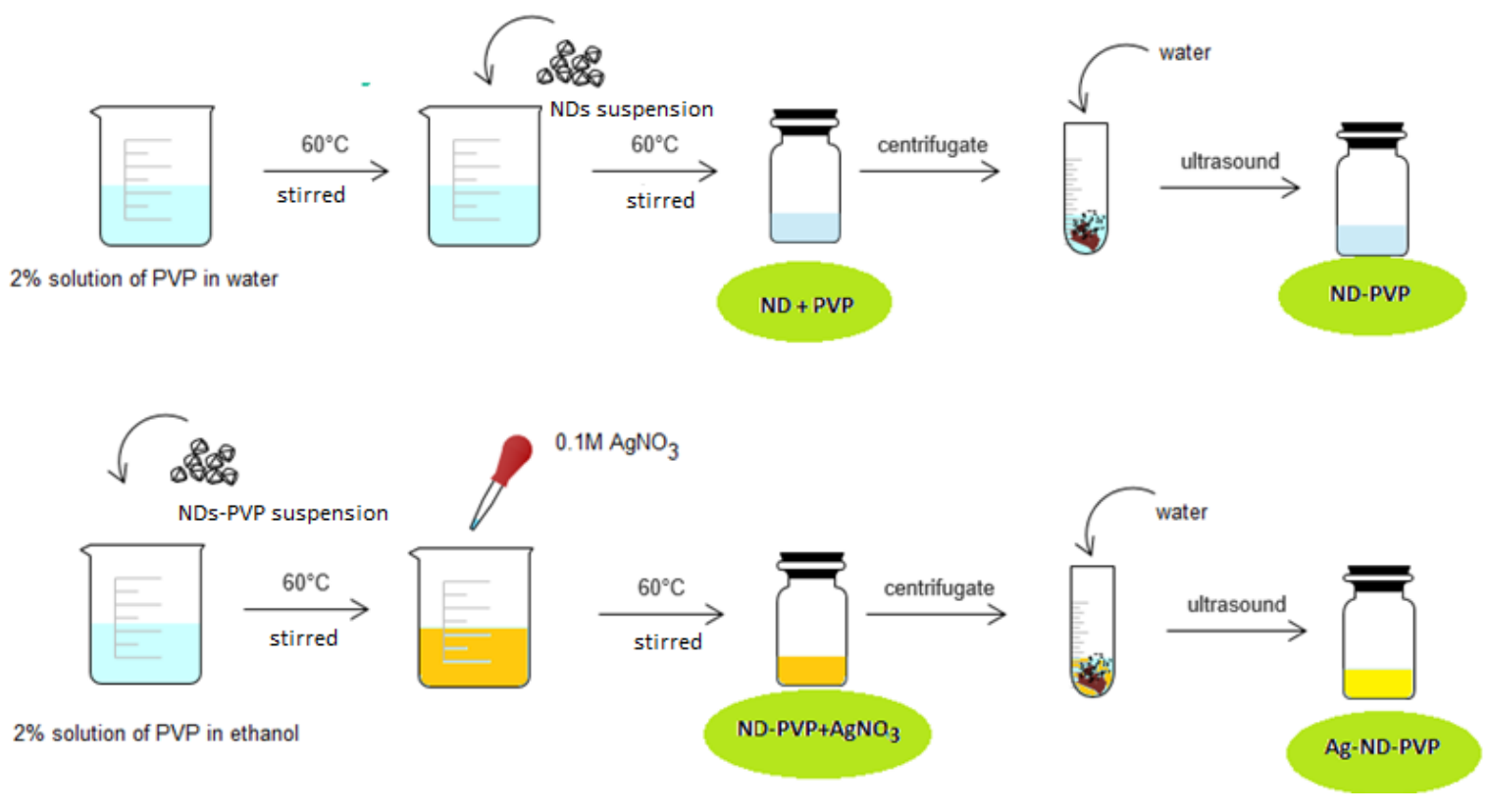

Figure 1 Scheme of sample preparation

Dynamic light scattering (DLS) was used for characterization of nanoparticles size distribution and zeta potential of the colloid solution, the measurements were performed by a Zetasizer Nano (Malvern Panalytical) equipped with a helium-neon laser $(633 \mathrm{~nm})$; the scattering angle was $173^{\circ}$.

For a colloidal stability test, $\mathrm{NaCl}$ was added to the nanodiamond solutions to a final concentration of $0.9 \%$ $\mathrm{w} / \mathrm{w}$. The evaluation was made by UV-Vis spectroscopy 2 hours after $\mathrm{NaCl}$ exposure. The measurements were done by Jasco V-730 UV-Vis double beam spectrophotometer with halogen and deuterium lamp as a light source.

The particle size and shape were observed with scanning electron microscope (SEM) and transmission electron microscope (TEM). SEM images were acquired by SEM MAIA 3 with ultra-high resolution in high vacuum (TESCAN). Samples for SEM were prepared by dropping the NDs solution onto a silicon substrate. 
TEM measurements were done by FEI Tecnai G2 20 with a LaB6 cathode at acceleration voltage $200 \mathrm{kV}$. The TEM is equipped with a CCD camera Olympus Veleta. Samples for TEM were prepared on carbon coated copper grid by immersion in ND colloid.

\section{RESULTS}

Nanoparticle size distribution was assessed by DLS measurements. Figure 2 shows histograms of nanoparticle size by volume. Size distribution shifted towards larger sizes after PVP coating, especially for MSY18. After decoration of the nanodiamonds with silver nanoparticles, the size histogram remains unchanged. During the preparation of the samples, the $\mathrm{pH}$ changes of the NDs solutions (Figure 3) and

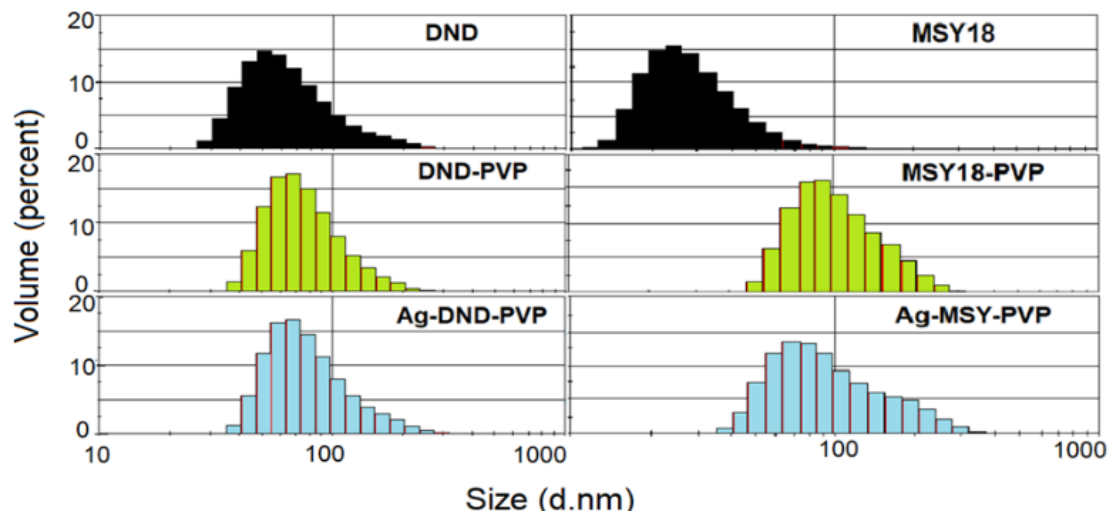

Figure 2 Size distribution histograms of prepared nanoparticles their zeta potentials (Figure 4) were studied. Zeta potential is an important indicator of stability of colloidal solutions. Suspensions with their zeta potential above $30 \mathrm{mV}$ or below $-30 \mathrm{mV}$ are considered stable. Colloidal stability is one of the critical factors for use of nanodiamonds. At pH 5, the colloidal solutions of annealed MSY particles sonicated in deionized water are well stable. The zeta potential of annealed DND nanoparticles is lower than in case of MSY $(-23 \mathrm{mV}$ for $\mathrm{pH}$ 6.5). The zeta potential is highly dependent on various chemical groups present on surface of the nanodiamonds. Carboxyl group is one such functional group and it determines the diamond surface charge [5]. Air oxidation of NDs at high temperature is responsible for creation of homogeneous layer of carboxyl groups on the surface of NDs. The difference between zeta potentials could be caused by varying methods of preparation and also by different particle sizes. After coating of NDs surface by PVP, the zeta potential for MSY nanodiamonds decreases while for DND it no change, up to a point where the value of zeta potential for both types of materials has stabilized at a similar number (see Figure 3). Carboxyl groups on the nanodiamond surface interact through bonding of hydrogen with pyridyl groups in PVP molecule [6]. PVP also acts as surface ligand to ensure dispersion of hybrid nanostructures in solution.

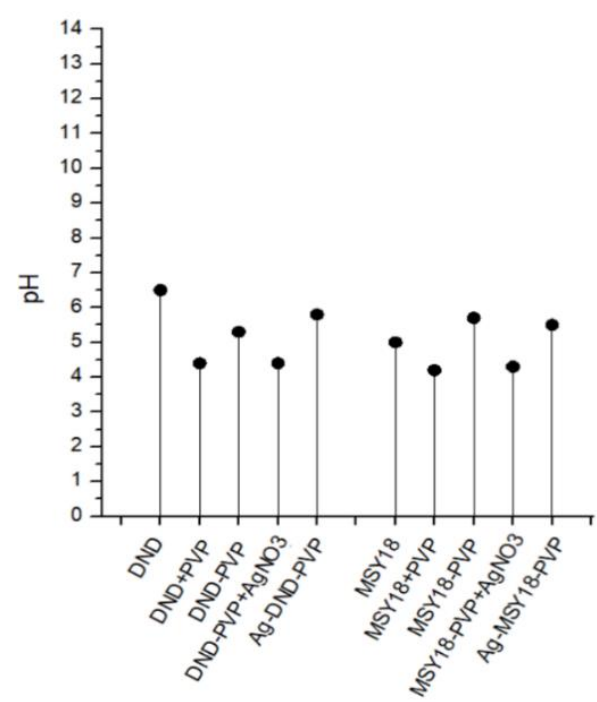

Figure $3 \mathrm{pH}$ changes during samples preparation

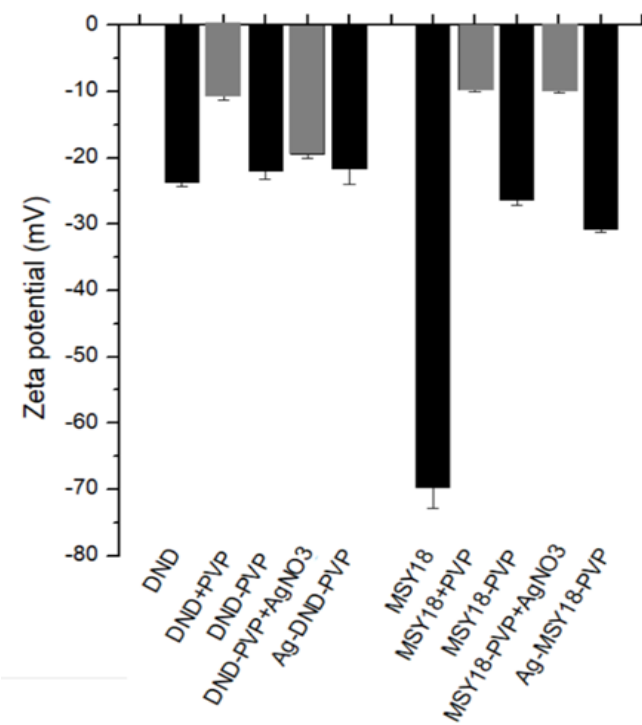

Figure 4 Zeta potential colloid solutions 
Coated nanodiamonds (MSY18-PVP and DND-PVP) prepared by this way were used for decoration with silver nanoparticles in situ (see Figure 1). Growth of Ag nanoparticles on NDs is performed in ethanol solution with PVP molecules acting as a reducing agent. The nitrogen atoms on the unbound pyridyl groups in PVP molecule coated nanodiamond surface possess strong affinity to silver ions and allow silver nanoparticle formation on the diamond surface. To detect changes in the shape and size of the agglomerates, the samples were observed by microscopy. The SEM images of coated nanodiamonds after decoration by silver nanoparticles are presented in Figures 5A for Ag-DND-PVP and 5B for Ag-MSY18-PVP. After decoration process, the surface of both nanodiamonds is covered with spherical silver nanoparticles. Backscattered-Electron (BSE) Imaging (right side of images) is used to detect contrast between areas with different chemical compositions. Since silver particles have higher atomic number, their electrons backscatter more strongly than those of lighter carbon, and thus appear brighter in the image. Bare NDs in colloids are electrostatically stabilized by zeta potential and thus have limited stability in solutions with ionic strength higher than $10^{-3} \mathrm{~mol} / \mathrm{L}$, the ND aggregate at higher ionic strengths [7]. However, SEM images of decorated nanoparticles did not confirm strong agglomeration effect in case of PVP-coated nanodiamonds; the agglomeration should usually occur with bare nanodiamonds during in situ reduction under similar experimental conditions. The silver nanoparticles have a diameter of about $10 \mathrm{~nm}$. Images were also taken by TEM microscopy to confirm and refine images of silver decorated nanoparticles (see Figures 6A for Ag-DND-PVP and 6B for Ag-MSY18-PVP).
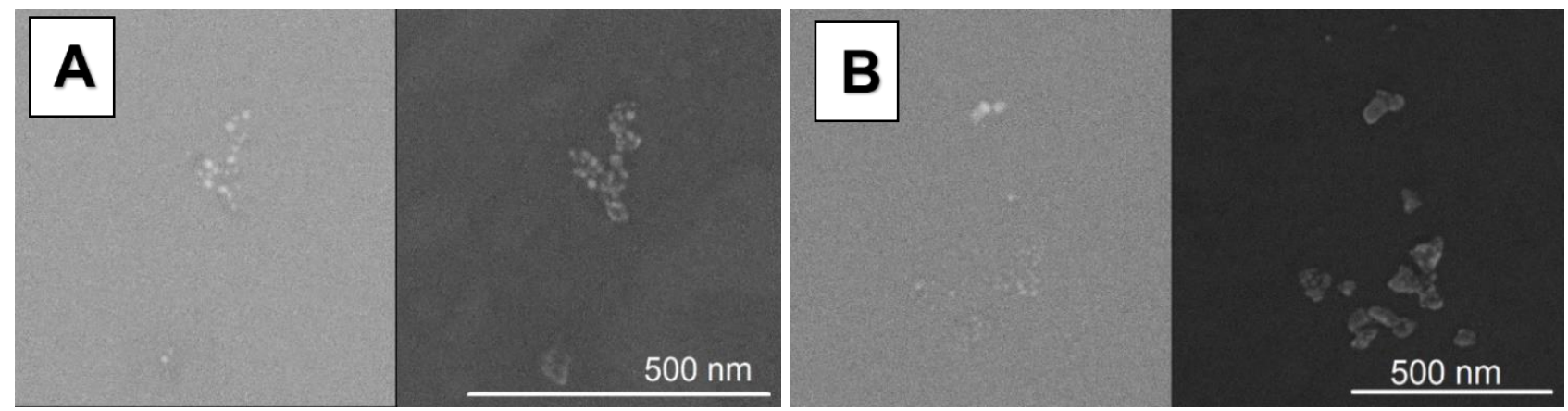

Figure 5 SEM images Ag-DND-PVP (A) and Ag-MSY18-PVP (B)
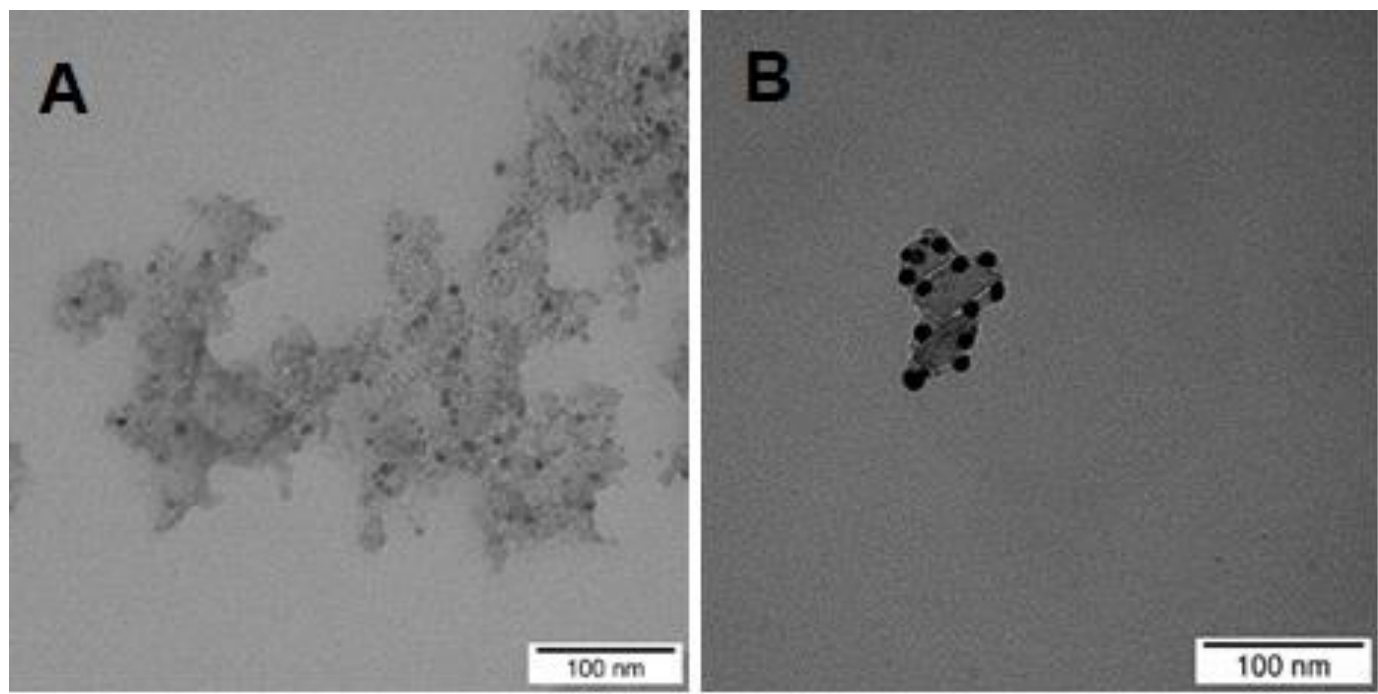

Figure 6 TEM images Ag-DND-PVP (A) and Ag-MSY18-PVP (B)

The DND nanodiamonds have form of tightly bound aggregates of particles called core agglutinates; this is typical for detonation-made nanoparticles. The agglutinate formation are attributed to facets-driven electrostatic interactions between individual DND particles and their liquid-phase sintering [8]. 
To determine the changes of stability of the studied nanoparticles in biological systems, $\mathrm{NaCl}$ was added to the aqueous suspension with pristine nanodiamonds, nanodiamonds in PVP and decorated nanodiamonds to a final $\mathrm{NaCl}$ concentration of $0.9 \%$ (same osmolarity as physiological solution). After only a few minutes, there was a visible turbidity of the bare nanodiamond suspension. Contrary, the suspension of NDs coated with PVP and NDs coated with PVP and decorated with silver nanoparticles did not show any visible changes after exposure to saline solution. All solutions were measured by UV-vis spectroscopy in the range of $350-800 \mathrm{~nm}$ to detect changes after the addition of $\mathrm{NaCl}$. From Figure 7 it is obvious that both types of bare nanodiamonds in saline solution lost their dispersibility and agglomerated, the samples coated with PVP retained their transparency indicating good dispersibility. UV-vis spectroscopy of the decorated nanocomposite solutions displayed the typical surface plasmon resonance peak for AgNPs ( $410 \mathrm{~nm}$ for nanoparticles with size of about $10 \mathrm{~nm}$ ) and the nanocomposite showed stability even in physiological saline solution in which bare NDs already aggregated [9].
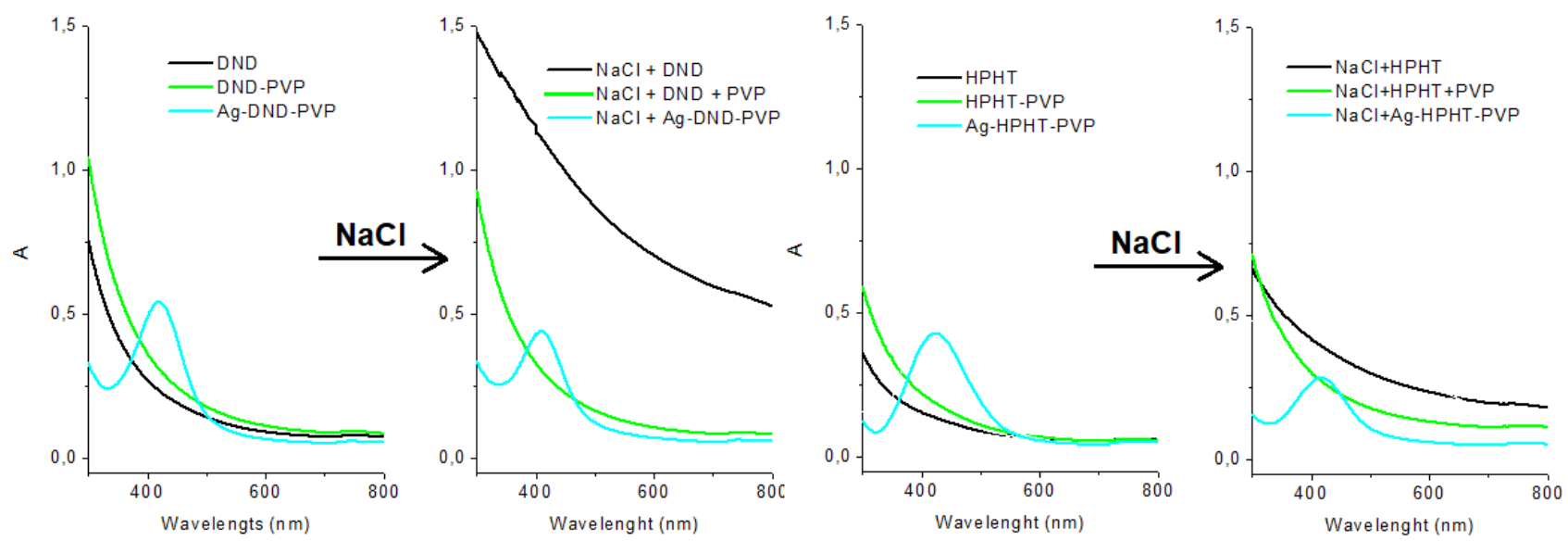

Figure 7 UV-Vis spectra of NDs solution before and after exposition of $\mathrm{NaCl}$

\section{CONCLUSION}

The nanodiamonds coated with a polyvinyl pyrrolidone showed improved stability by preventing ND agglomeration even in physiological solution. The final zeta potential of coated nanodiamonds solution was $-25 \mathrm{mV}$. By the polymer coating, we also achieved the attachment of AgNPs onto the ND surface. The final Ag-NDs composite material also showed good stability in deionized water and $0.9 \% \mathrm{NaCl}$. In this context, the PVP-coating of nanodiamonds seems as a promising way how to stabilize not only the HPHT, but also the detonation nanodiamonds in demanding biological environments and how to achieve controlled decoration of NDs by metal nanoparticles.

\section{ACKNOWLEDGEMENTS}

The work is supported by Operational Programme Research, Development and Education financed by European Structural and Investment Funds and the Czech Ministry of Education, Youth and Sports (Project No. SOLID21 CZ.02.1.01/0.0/0.0/16_019/0000760). We also acknowledge use of the CzechNanoLab research infrastructure supported by the MEYS (LM2018110).

\section{REFERENCES}

[1] MOCHALIN, V. N., SHENDEROVA, O., HO, D., GOGOTSI, Y. The properties and applications of nanodiamonds. Nat. Nanotechnol. 2012, vol. 7, pp. 11-23. 
[2] SCHRAND, A. M., HUANG, H., CARLSON, C., SCHLAGER, J. J., ŌSAWA, E., HUSSAIN, S. M., DAI, L. Are Diamond Nanoparticles Cytotoxic? J. Phys. Chem. B. 2007, vol. 111, pp. 2-7.

[3] NUNN, N., TORELLI, M., MCGUIRE, G., SHENDEROVA, O. Nanodiamond: A high impact nanomaterial. Curr. Opin. Solid State Mater. Sci. 2017, vol. 21, pp. 1-9.

[4] ZHANG, Y., RHEE, K. Y., HUI, D., PARK, S.-J. A critical review of nanodiamond based nanocomposites: Synthesis, properties and applications. Compos. Part B Eng. 2018, vol. 143, pp. 19-27.

[5] ZHUKOV, A. N., GAREEVA, F. R., ALEKSENSKII, A. E., VUL', A. Ya. Surface charge of detonation nanodiamond particles in aqueous solutions of simple 1: 1 Electrolytes. Colloid J. 2010, vol. 72, pp. 640-646.

[6] GONG, J., STEINSULTZ, N., OUYANG, M. Nanodiamond-based nanostructures for coupling nitrogen-vacancy centres to metal nanoparticles and semiconductor quantum dots. Nat. Commun. 2016, vol. 7, p. 11820.

[7] YOSHIKAWA, T., ZUERBIG, V., GAO, F., HOFFMANN, R., NEBEL, C. E., AMBACHER, O., LEBEDEV, V. Appropriate Salt Concentration of Nanodiamond Colloids for Electrostatic Self-Assembly Seeding of Monosized Individual Diamond Nanoparticles on Silicon Dioxide Surfaces. Langmuir. 2015, vol. 31, pp. 5319-5325.

[8] STEHLIK, S., HENYCH, J., STENCLOVA, P., KRAL, R., ZEMENOVA, P., PANGRAC, J., VANEK, O., KROMKA, A., REZEK, B. Size and nitrogen inhomogeneity in detonation and laser synthesized primary nanodiamond particles revealed via salt-assisted deaggregation. Carbon. 2021, vol. 171, pp. 230-239.

[9] BRADAC, C., RASTOGI, I. D., CORDINA, N. M., GARCIA-BENNETT, A., BROWN, L. J. Influence of surface composition on the colloidal stability of ultra-small detonation nanodiamonds in biological media. Diam. Relat. Mater. 2018, vol. 83, pp. 38-45. 